\title{
Proportional representation
}

\section{The journal publishes papers from a very broad geographical catchment, and we invite peer referees from among the world's best genetics researchers in order to attract and publish papers of a uniformly high standard. We need to do more to recruit outstanding referees from under-represented regions.}

ditors make lab visits and meet authors at conferences not only to learn about research that is ready to be published in the journal, but also to hear critical comments from authors about research published in the journal. These conversations are often a good way to identify potential new peer referees who will be committed to raising the standard of the papers we publish. Because we are full-time editors and we do not have a formal editorial board of advisors, constant feedback about our own decisions and the advice we receive from referees helps shape our ever-improving criteria for accepting manuscripts. New contacts also allow us move the focus and expand the scope of the journal to take advantage of fruitful fields of research as they open up.

We are proud that genetics and genomics are among the most global fields of science, bringing us diverse projects from many countries as well as international collaborative research-more than half of the research articles we publish are the products of more than one country. We are also concerned about understanding disparities by country and region in the ratio of submissions to successful publications. Yes, we decide editorially which submissions to send to review (currently about 20\%) and when the papers are ready to publish, but the advice the authors and we receive during review is key. So is quality. For us simply to send more papers from less successful regions out for review is not a sustainable way to achieve more publications.

Many of the papers we receive and return to authors are not within the scope of the journal-that is, they are not genetics. Authors who practice some self-selection-choosing the journal on the basis of recently published benchmark papers and our declared fields of interest rather than just by our reputation for excellence or any related metrics-have the most success. Similarly, it is our declared intent to publish genetics and genomics at the same level as Nature, so it should be obvious that our standards are no lower than those of our sister journal. In particular, authors of successful papers are those who are more careful to separate their experiments into discrete steps of hypothesis generation and validation and who are skeptical about their own results.

The editors find that presubmission enquiries (read by all the editors and returned to authors within a couple of working days) are a particularly good means for authors to gauge our standards and our level of confidence in the potential success of their work at peer review. We can also advise on how many papers to divide the work into, how much work to put into any one paper, and any experiments currently considered essential in a particular subfield. These enquiries may involve sending as little as an abstract, or possibly some data figures or even a complete manuscript, and they should be submitted via our online database, since individual editors are likely to be making lab visits and may not immediately respond by email. The entry in the database does not commit your paper to one journal (and you may enquire of more than one journal), but it provides a record and a reminder to us when your paper arrives.

Unsuccessful submissions should provide some indication of the journal's decision-making process and the criteria we apply; if these are not clear, please ask the manuscript editor for clarification, as misunderstandings can occasionally result in lengthy and usually unsuccessful appeals against our decisions. Appeals in which the authors offer to add data and more analysis are more likely to be successful.

Although we need to work with "calibrated" expert referees who can consistently provide us with the best advice, we are constantly adding referees to the papers we send to review. This process ensures that new referees see more excellent papers, understand the standards applied by referees and editors, and make their own advice clearer and more useful. We believe that researchers who act as referees also benefit because experience gained while reviewing other people's papers will make them more likely to have success with their own papers here and at all high-quality journals. Regional discrepancies will be progressively corrected as we recruit referees. Most importantly for us, this journal will incorporate their advice and standards and continue to grow. 